\title{
INTEGRAL observations of the 2007 outburst of the Be transient SAX J2103.5+4545
}

\author{
L. Ducci ${ }^{\star a b}$, L. Sidoli ${ }^{b}$, A. Paizis ${ }^{b}$, S. Mereghetti ${ }^{b}$, P. M. Pizzochero ${ }^{c}$ \\ ${ }^{a}$ Dipartimento di Fisica e Matematica, Università degli Studi dell'Insubria, \\ Via Valleggio 11, I-22100 Como, Italy \\ ${ }^{b}$ INAF, Istituto di Astrofisica Spaziale e Fisica Cosmica, \\ Via E. Bassini 15, I-20133 Milano, Italy \\ ${ }^{c}$ Dipartimento di Fisica, Università degli Studi di Milano, \\ Via Celoria 16, I-20133 Milano, Italy \\ E-mail: lorenzodiasf-milano.inaf.it
}

We performed a detailed study of the 2007 outburst of the $352 \mathrm{~s}$ pulsar and Be/X-ray transient SAX J2103.5+4545 with INTEGRAL, to study its spectral and temporal properties during the evolution of the outburst. SAX J2103.5+4545 was observed with IBIS/ISGRI from 25 to 27 April 2007 and from 6 to 8 May 2007. The $20-100 \mathrm{keV}$ spectrum is well described by a bremsstrahlung model with a temperature $\mathrm{kT} \sim 24 \mathrm{keV}$. The pulse profiles are variable with time and energy. A spin-up of $\dot{P}_{\text {spin-up }}=(-3.4 \pm 0.4) \times 10^{-7} \mathrm{~s} \mathrm{~s}^{-1}$ is measured during the outburst. Instead, a spin-down of $\dot{P}_{\text {spin-down }}=(5.5 \pm 0.4) \times 10^{-9} \mathrm{~s} \mathrm{~s}^{-1}$ is observed between the 2007 outburst reported here and the previous one occurred in December 2004. This is the largest spindown measured for SAX J2103.5+4545 since its discovery. We estimate a neutron star magnetic field in the range $(1.6-3) \times 10^{13} \mathrm{G}$ using the Ghosh \& Lamb torque model.

7th INTEGRAL Workshop

September 8-11 2008

Copenhagen, Denmark

\footnotetext{
* Speaker.
} 


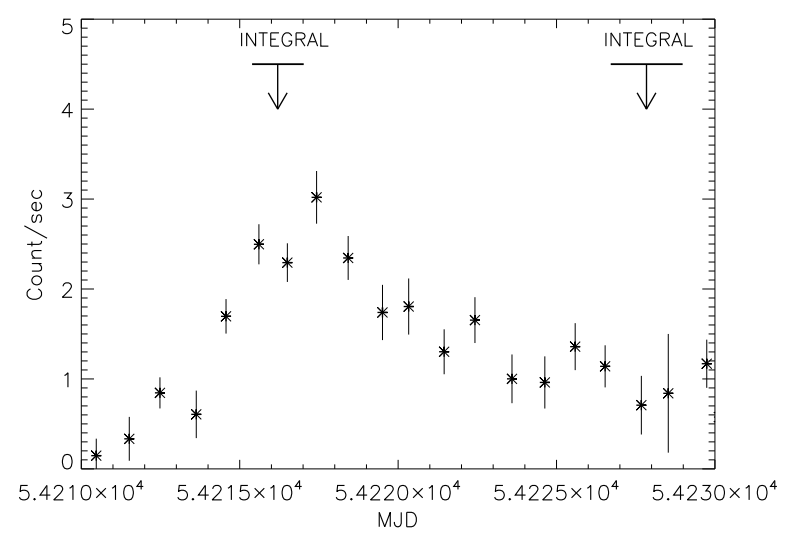

Figure 1: $R X T E / A S M$ light-curve of SAX J2103.5+4545 in the 2-10 keV energy range (54210 - 54230 MJD, April 20 - May 10 2007). The arrows indicate the time interval covered by the INTEGRAL observations.

\section{Introduction}

SAX J2103.5+4545 is a transient Be/X-ray binary with an orbital period of 12.67 days [6] hosting a pulsar with a pulse period of $352.2 \mathrm{~s}$ [ []]. It was discovered during an outburst in February 1997 [11], and since then numerous type I and type II outbursts have been observed [6]. The optical counterpart is a B0Ve star with highly variable $\mathrm{H} \alpha$ emission, located at a distance of $6.5 \pm 0.9 \mathrm{kpc}$ [14]. The position of SAX J2103.5+4545 in the spin period versus orbital period diagram deviates from the correlation of HMXB with Be star and falls in the region typical of wind-fed HMXB with OB supergiant companions. The X-ray energy spectrum in the 5-200 keV band is described by a power-law with exponential cut-off, with photon index $\Gamma=1.53$, cut-off energy $E_{c}=19 \mathrm{keV}$ and e-folding energy $E_{F}=32 \mathrm{keV}$. No cyclotron lines have been observed [16]. A soft blackbody component with temperature $\mathrm{kT} \sim 1.9 \mathrm{keV}$ was observed with XMM-Newton [12], and an iron emission line at $\sim 6.42 \mathrm{keV}$ was observed with RXTE [3]. SAX J2103.5+4545 has shown numerous spin-up phases during its outbursts. A correlation between X-ray flux and spin-up rate has been found [2] which can be explained with the formation of an accretion disk.

\section{Observations and data analysis}

SAX J2103.5+4545 underwent an outburst in April-May 2007 [9]. This outburst was observed by the IBIS/ISGRI instrument on 25-27 April 2007 (54215.39-54217.02 MJD) and on 6-8 May 2007 (54226.71 - 54228.98 MJD).

Here we report the data analysis of the source field observed with IBIS-ISGRI. The Off-line Scientific Analysis (OSA 6.0) software was used. We have divided this observations in 2 groups (Table 1) which are marked in Figure 1, where the 2-10 keV light-curve of the 2007 outburst observed by RXTE/ASM is reported.

We extracted two spectra of SAX J2103.5+4545 in the energy range $22-100 \mathrm{keV}$, in the time intervals 25-27 April 2007 and 6-8 May 2007 (Table 1). A systematic uncertainty of 1\% was added quadratically to the statistical errors to account for uncertainties in the instrumental response. 
Table 1: The two groups of IBIS/ISGRI observations of SAX J2103.5+4545 used in this work.

\begin{tabular}{lcccc}
\hline \hline Obs. & Time interval & $\begin{array}{c}\text { Start Time } \\
(\mathrm{MJD})\end{array}$ & $\begin{array}{c}\text { End Time } \\
(\mathrm{MJD})\end{array}$ & $\begin{array}{c}\text { Exposure time } \\
(\mathrm{ks})\end{array}$ \\
\hline 1 & 25-27 April 2007 & 54215.39 & 54217.02 & 129 \\
2 & 6-8 May 2007 & 54226.71 & 54228.98 & 169 \\
\hline
\end{tabular}

We performed a timing analysis in the range $15-60 \mathrm{keV}$, where the best signal to noise ratio is obtained. We corrected the arrival times to the Solar System barycenter and for Doppler delay due to the pulsar orbital motion. The correction for the Doppler delay can be written as:

$$
\delta t=x \sin l-\frac{3}{2} x e \sin \omega-\frac{1}{2} x e \sin (\omega-2 l)
$$

where $x=a_{x} \sin (i) / c$ is the light-travel time for the projected semi-major axis $a_{x}, i$ is the inclination angle, $e$ is the eccentricity and $\omega$ is the longitude of periastron [8]. $l$ is the mean orbital longitude at time $t$ which is given by:

$$
l=2 \pi \frac{\left(t-T_{\pi / 2}\right)}{P_{o r b}}+\frac{\pi}{2}
$$

where $T_{\pi / 2}$ is the epoch when the mean orbital longitude is equal to $\pi / 2$ and $P_{\text {orb }}$ is the orbital period. To correct the arrival times, we have assumed the most recent orbital parameters obtained by Camero Arranz et al. (2007) [6]. An epoch folding technique was used to measure the pulse period [13].

\section{Results}

\subsection{Spectral analysis}

We have fitted the $22-100 \mathrm{keV}$ spectra with several simple models and found that the best $\chi^{2}$ value is given by a bremsstrahlung model with a temperature $k T \approx 24 \mathrm{keV}$ for both spectra (see Figure 2 for energy spectra, and Table 2 for parameters).

The average flux (20-100 keV) obtained from the spectrum in the time interval 25-27 April 2007 is $F_{x}=\left(1.68_{-0.07}^{+0.09}\right) \times 10^{-9} \mathrm{erg} \mathrm{cm}^{-2} \mathrm{~s}^{-1}$, while on 6-8 May 2007 the flux is $F_{x}=\left(5.0_{-0.06}^{+0.06}\right) \times$ $10^{-10} \mathrm{erg} \mathrm{cm}^{-2} \mathrm{~s}^{-1}$, translating into a luminosity $L_{x}=(8.5 \pm 2.5) \times 10^{36} \mathrm{erg} \mathrm{s}^{-1}(25-27$ April $2007)$ and $L_{x}=(2.5 \pm 0.7) \times 10^{36} \mathrm{erg} \mathrm{s}^{-1}(6-8$ May 2007) for a distance $d=6.5 \pm 0.9 \mathrm{kpc}$. These luminosities are similar to those measured during previous outbursts ([16], [1]).

\subsection{Timing analysis}

We measured the pulse period and the associated uncertainty for each of the two data sets reported in Table 1 by means of an epoch folding technique, obtaining the values reported in Table 3. The average spin-up rate between $P_{1}$ and $P_{2}$ is $\dot{P}_{\text {spin-up }}=(-3.4 \pm 0.4) \times 10^{-7} \mathrm{~s} \mathrm{~s}^{-1}$ The average flux during this spin-up is $\bar{F}_{x}=3.6 \times 10^{-9} \mathrm{erg} \mathrm{cm}^{-2} \mathrm{~s}^{-1}$ in the energy range $1-200 \mathrm{keV}$.

SAX J2103.5+4545 has shown a pulse period decrease since 1997, occasionally interrupted by spin-down intervals, corresponding to low accretion rates. ([16], [1]). In particular, the pulse 

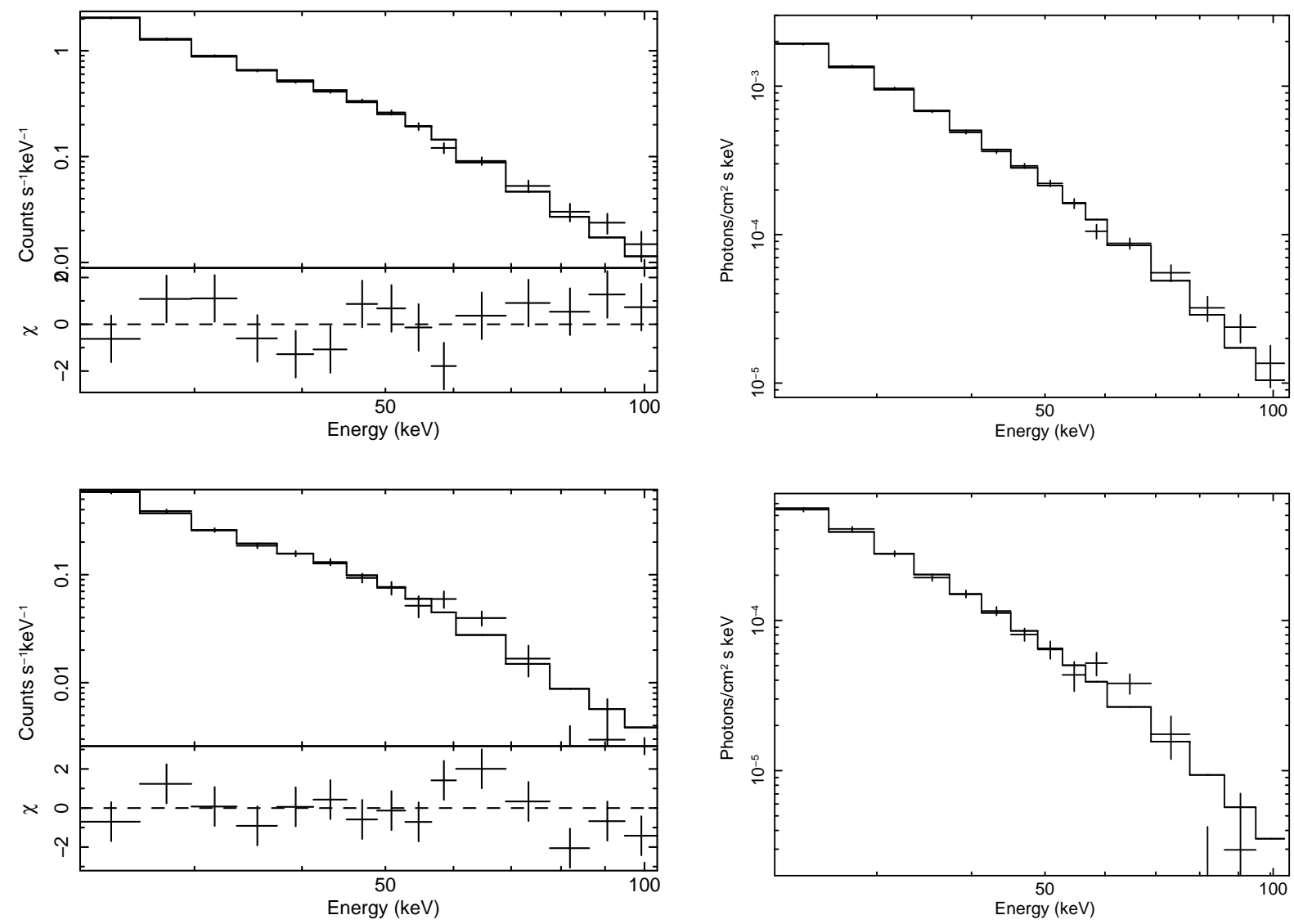

Figure 2: ISGRI SAX J2103.5+4545 spectra in the energy range 22-100 keV, on 25-27 April 2007 (Top) and on 6-8 May 2007 (Bottom). Left panels: counts spectra, best-fit bremsstrahlung spectra and residuals (in units of standard deviations); Right panels: photon spectra together with the best-fit model.

Table 2: Best fit parameters of the two average spectra of SAX J2103.5+4545 with a bremsstrahlung model.

\begin{tabular}{lcccc}
\hline \hline Time interval & $\begin{array}{c}\mathrm{kT} \\
(\mathrm{keV})\end{array}$ & $\begin{array}{c}\chi_{v}^{2} \\
\text { for 13 (d.o.f.) }\end{array}$ & $\begin{array}{c}\text { Flux }(20-40 \mathrm{keV}) \\
10^{-9} \mathrm{erg} \mathrm{cm}^{-2} \mathrm{~s}^{-1}\end{array}$ & $\begin{array}{c}\text { Flux }(20-100 \mathrm{keV}) \\
10^{-9} \mathrm{erg} \mathrm{cm}^{-2} \mathrm{~s}^{-1}\end{array}$ \\
\hline 25-27 April 2007 & $23.4_{-0.7}^{+0.8}$ & 1.06 & $1.11_{-0.03}^{+0.04}$ & $1.68_{-0.07}^{+0.09}$ \\
& $24_{-2}^{+2}$ & 1.29 & $0.32 \pm 0.03$ & $0.50 \pm 0.06$ \\
\hline
\end{tabular}

Table 3: Pulse periods of SAX J2103.5+4545.

\begin{tabular}{lcc}
\hline \hline Name & Time interval & $P_{\text {spin }}(\mathrm{s})$ \\
\hline$P_{1}$ & 25-27 April 2007 & $352.725 \pm 0.004$ \\
$P_{2}$ & 6-8 May 2007 & $352.382 \pm 0.004$ \\
\hline
\end{tabular}




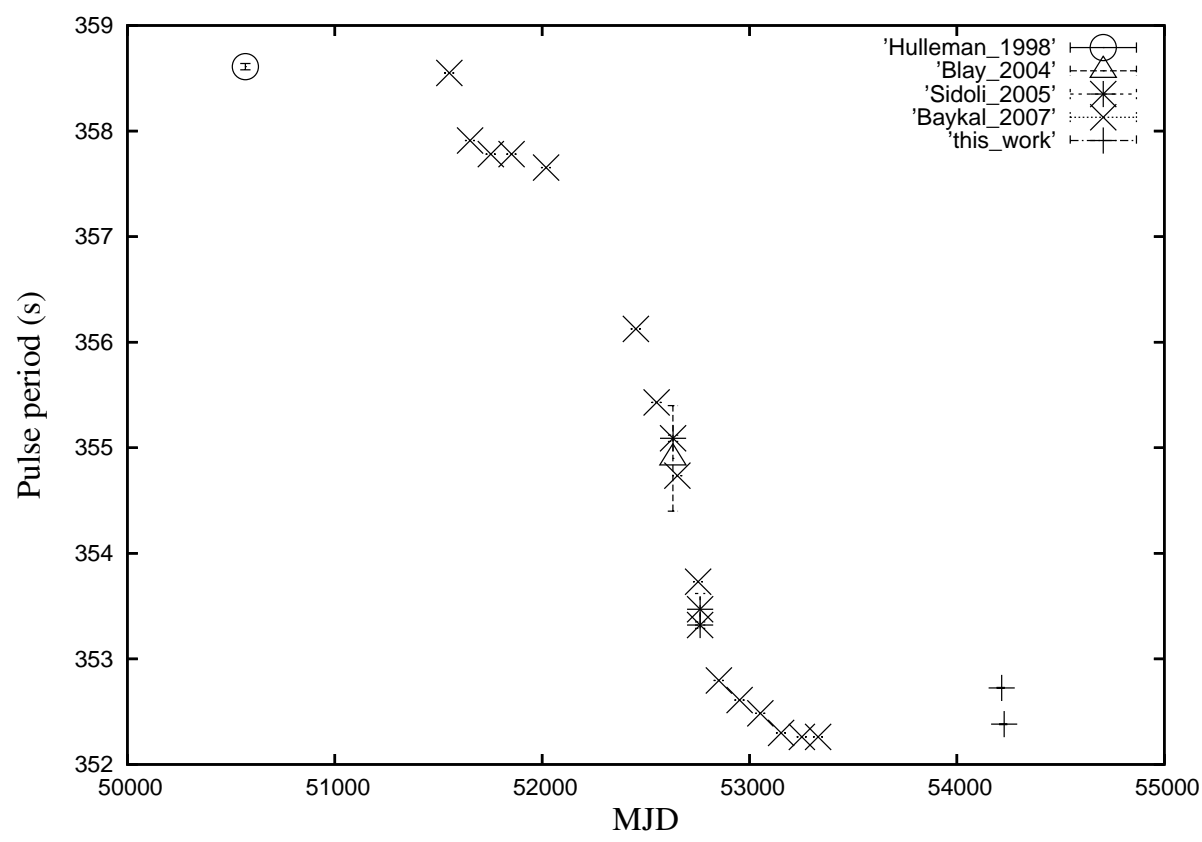

Figure 3: Pulse period history of SAX J2103.5+4545. The pulse period values are taken by BeppoSAX [11], INTEGRAL [4], [16], and RXTE [1].

period history (Figure 3) shows a spin-down between December 2004 (53340 \pm 10 MJD) where $P_{\text {Baykal } 2007}=352.31 \pm 0.01 \mathrm{~s}$ [1], and the last outburst (April - May 2007, see Table 1), when we measured $P_{1}=352.725 \pm 0.004$ s. Between these two outbursts SAX J2103.5+4545 was in a low luminosity state. We measured the upper limit flux of SAX J2103.5+4545 during this low luminosity state from December 2004 to April 2007 using RXTE/ASM data. We found $F<2 \times$ $10^{-11} \mathrm{erg} \mathrm{cm}^{-2} \mathrm{~s}^{-1}$ in the energy range $2-10 \mathrm{keV}$. The average pulse period derivative from December 2004 to April 2007 is $\dot{P}_{\text {spin-down }}=(5.5 \pm 0.4) \times 10^{-9} \mathrm{~s} \mathrm{~s}^{-1}$.

For each of the 2 data sets reported in Table 11, we have obtained the pulse profiles for the energy bands $15-60 \mathrm{keV}, 15-40 \mathrm{keV}$ and $40-60 \mathrm{keV}$. The pulse profiles are binned into 20 phase bins (zero phase at $51000.000 \mathrm{MJD}$; see Figure 4 . We have found a temporal variability for the pulse profiles, as already observed during previous outbursts of this source ([6], [16]).

\section{Discussion and Conclusions}

SAX J2103.5+4545 is a Be/X-ray binary with transient X-ray emission, fed by the wind of the Be companion. In previous outbursts a correlation between X-ray flux and spin-up rate was found which can be explained with the formation of an accretion disk [2].

When a neutron star is fed by an accretion disk, the material which flows towards the neutron star magnetic poles produces X-ray luminosity and at the same time a torque on the rotating neutron stars mediated by its magnetosphere. The angular momentum of the infalling matter is transferred to the pulsar at the magnetospheric boundary [10]. The Ghosh and Lamb model assumes a magnetically-threaded disk allowing not only spin-up, but also spin-down, which occurs when the magnetic and corotation radii are comparable and the luminosity is low. Ghosh and Lamb 
Obs. 1

Obs. 2

$15-40 \mathrm{keV}$ :
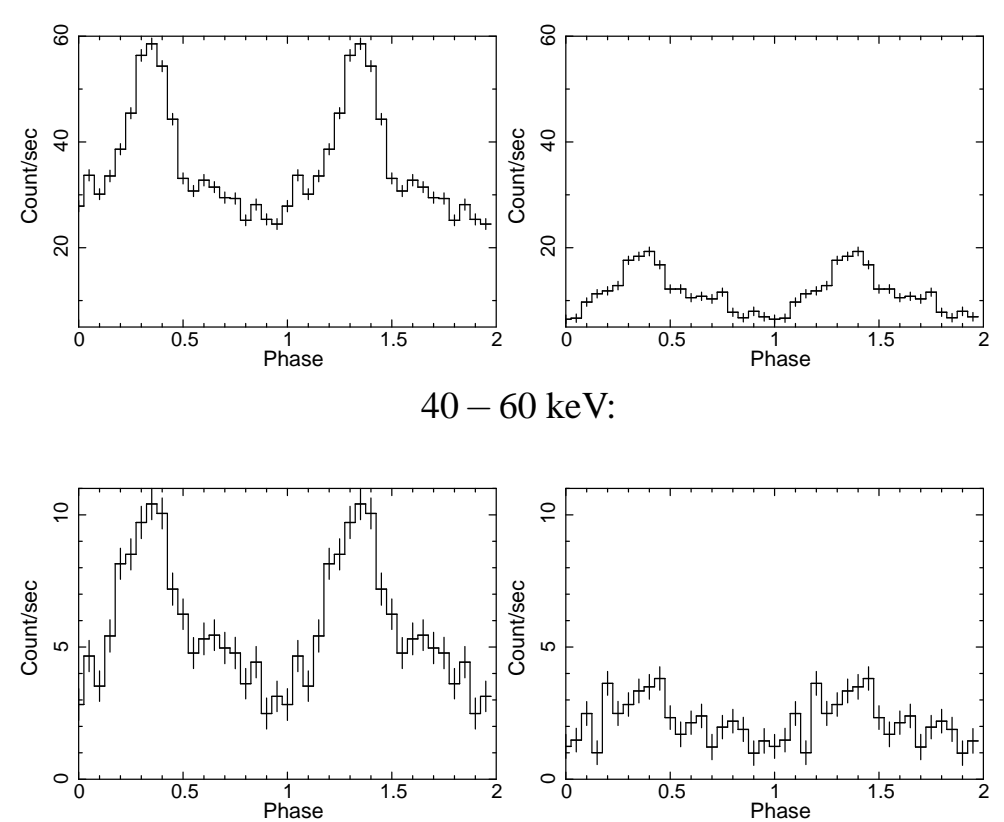

Figure 4: Evolution of SAX J2103.5+4545 pulse profiles for different time intervals and for two energy bands. From top to bottom: $15-40 \mathrm{keV}, 40-60 \mathrm{keV}$. From left to right: Obs. 1, Obs. 2 (see Table 3).

predicted the following correlation between the pulse period derivative and the X-ray luminosity:

$$
\dot{P}=-5.0 \times 10^{-5} \mu_{30}^{2 / 7} n\left(\omega_{s}\right) R_{6}^{6 / 7} I_{45}^{-1}\left(P L_{37}^{3 / 7}\right)^{2}\left(\frac{M_{N S}}{M_{\odot}}\right)^{-3 / 7} \mathrm{~s} \mathrm{yr}^{-1}
$$

where $\mu_{30}$ is the neutron star magnetic dipole moment in the disk plane $\left(\mu=B R^{3}\right)$ in units of $10^{30} \mathrm{G} \mathrm{cm}^{3}, R_{6}$ is the stellar radius in units of $10^{6} \mathrm{~cm}, M_{N S}$ is the mass of the neutron star in grams, $I_{45}$ is the moment of inertia in units of $10^{45} \mathrm{~g} \mathrm{~cm}^{2}, P$ is the pulse period, $L_{37}$ is the accretion luminosity in units of $10^{37} \mathrm{erg} \mathrm{s}^{-1}$. We have considered the dimensionless torque obtained by Ghosh \& Lamb (1979) [10]:

$$
n\left(\omega_{s}\right) \approx 1.39 \frac{1-\omega_{s}\left[4.03\left(1-\omega_{s}\right)^{0.173}-0.878\right]}{1-\omega_{s}}
$$

where $\omega_{s}$ is called fastness parameter and is defined as:

$$
\omega_{s} \equiv \frac{\Omega_{s}}{\Omega_{K}\left(r_{0}\right)}=\left(\frac{r_{0}}{R_{c}}\right)^{3 / 2}
$$

where $\Omega_{s}$ is the angular velocity of the star, $\Omega_{K}\left(r_{0}\right)=\left(G M_{N S} / r_{0}^{3}\right)^{1 / 2}$ is the Keplerian angular velocity at $r_{0}$, the radius which divides the outer part of the accretion disk, where the velocity is keplerian, from the inner part, where the plasma corotates with the star [10]; $R_{c}=\left(G M_{N S} / \Omega_{s}^{2}\right)^{1 / 3}$ is the corotation radius of the star. Therefore, during an outburst, a variation in the accretion rate can modify the pulse period derivative and at the same time the X-ray luminosity. In this 


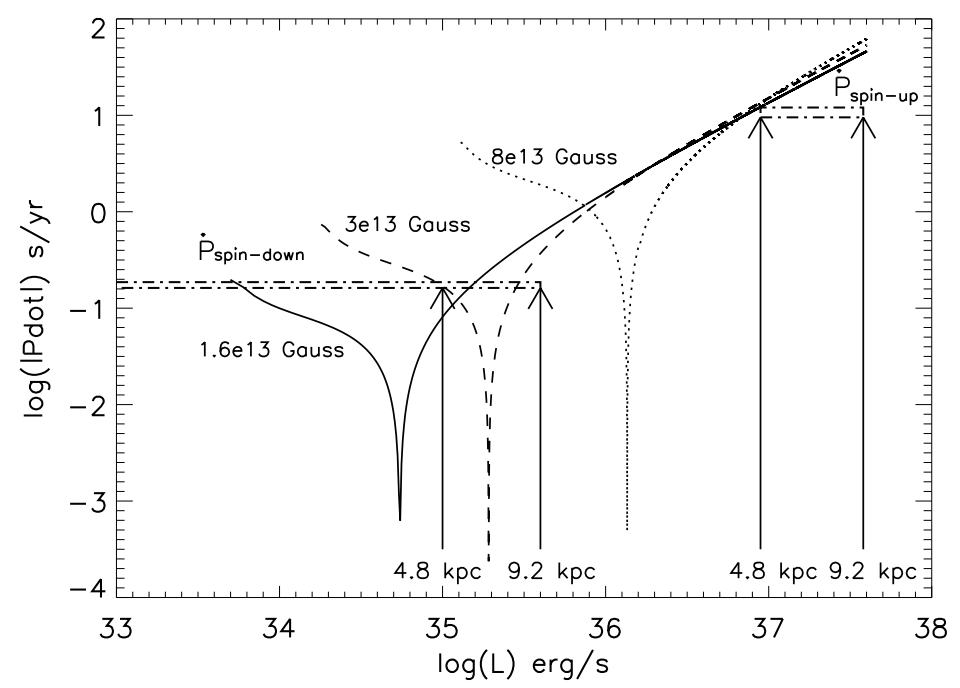

Figure 5: Comparison between the observed spin-up and spin-down rate and the theoretical relation between $\dot{P}$ and the source luminosity. The box labeled with $\dot{P}_{\text {spin-up }}$ indicates the spin-up rate measured during the time interval 25 April - 8 May 2007, the box labeled with $\dot{P}_{\text {spin-down }}$ indicates the spin-down rate measured during the time interval December 2004 - April 2007. The fit with theoretical curves is obtained for a distance $\sim 4.8 \mathrm{kpc}$ and a magnetic field $B=1.6 \times 10^{13} \mathrm{G}$ (solid line) and $B=3 \times 10^{13} \mathrm{G}$ (dashed line). The uncertainties of $\dot{P}_{\text {spin-up }}, \dot{P}_{\text {spin-down }}$ and $L_{x}$ are given by the pulse period derivative uncertainties and by the uncertainty of the source distance.

framework it is possible to determine the magnetic field of SAX J2103.5+4545. Using equations (4.1) to (4.3), we assumed $M_{N S}=1.4 \mathrm{M}_{\odot}, R_{N S}=10 \mathrm{~km}$, an average pulse period $\bar{P}=352.5 \mathrm{~s}$, $\bar{F}_{x}=3.6 \times 10^{-9} \mathrm{erg} \mathrm{cm}^{-2} \mathrm{~s}^{-1}$ as average flux during the spin-up, and the upper limit flux during the spin-down. We found a magnetic field of $(1.6-3) \times 10^{13} \mathrm{G}$ for a distance of $\sim 4.8 \mathrm{kpc}$. This distance is consistent with what found by Reig et al. (2004), if the error in the absolute magnitude found by Vacca et al. (1996) [17] is included. These results are also consistent with what previously obtained by Baykal et al. (2007) [1]. In Figure (5) we compare the spin-up and spin-down rate observed with INTEGRAL with the theoretical relation between $\dot{P}$ and the source luminosity for 3 different magnetic fields of the neutron star. Vertical lines mark the source luminosities for $d=4.8 \mathrm{kpc}$ and $d=9.2 \mathrm{kpc}$.

During the low luminosity state (December 2004 - April 2007) the upper-limit to the luminosity is $L_{x}<5 \times 10^{34} \mathrm{erg} \mathrm{s}^{-1}(2-10 \mathrm{keV}, d=4.5 \mathrm{kpc})$. This low luminosity cannot be explained with the high-density $\left(\dot{M}=10^{-7} \mathrm{M}_{\odot} \mathrm{yr}^{-1}\right)$ wind of the circumstellar disk around the Be star. From optical observation during low luminosity states, SAX J2103.5+4545 shows $H \alpha$ in absorption, which is interpreted as the loss of the circumstellar disk ([14], [15]). In this case, SAX J2103.5+4545 could accrete from the low-density wind of the polar regions. The mass loss rate out of circumstellar disk is $10^{-8}-10^{-9} \mathrm{M}_{\odot} \mathrm{yr}^{-1}$, the velocity law is $v=v_{\infty}\left(1-R_{B e} / r\right)^{\beta}$, with terminal velocity $v_{\infty} \approx 600-1800 \mathrm{~km} \mathrm{~s}^{-1}, \beta \approx 1$ ([19], [18]). Assuming the SAX J2103.5+4545 parameters found by Camero Arranz et al. 2007 [6], and assuming $M_{B e}=20 \mathrm{M}_{\odot}, R_{N S}=10 \mathrm{~km}, M_{N S}=1.4 \mathrm{M}_{\odot}$ and 
$R_{B e}=8 \mathrm{R}_{\odot}$ ([14], [15]), we find that the expected luminosity in the Bondi-Hoyle accretion theory ([⿰], [18]) is $L_{x} \leq 10^{34} \mathrm{erg} \mathrm{s}^{-1}$. If we consider the pulsar magnetic field of $3 \times 10^{13} \mathrm{G}$, calculated above, a wind mass loss rate of $8 \times 10^{-9} \mathrm{M}_{\odot} \mathrm{yr}^{-1}$, and $v_{\infty} \approx 600 \mathrm{~km} \mathrm{~s}^{-1}$, the centrifugal inibition of accretion produces an expected luminosity $L_{x}=\frac{G M_{N S}}{R_{m}} \dot{M} \leq 10^{31} \mathrm{erg} \mathrm{s}^{-1}$, where $R_{m}$ is the magnetospheric radius [7]. These expected luminosities agree with the measured upper-limit, and with the observed spin-down rate $\dot{P}_{\text {spin-down }}=(5.5 \pm 0.4) \times 10^{-9} \mathrm{~s} \mathrm{~s}^{-1}$.

\section{Acknowledgments}

LS, AP and SM acknowledge the Italian Space Agency financial and programmatic support via contract I/008/07/0. The autors thank the INTEGRAL Science Data Center shift team, in particular V. Beckmann, S. Shaw and N. Produit for efficiently sharing the Quick Look Analysis results of SAX J2103.5+4545.

\section{References}

[1] Baykal, A., Inam, S. Ç., Stark, M. J., et al. 2007, MNRAS, 374, 1108

[2] Baykal, A., Stark, M. J., Swank, J. 2000, ApJ, 544, L129

[3] Baykal, A., Stark, M. J., Swank, J. H., 2002, ApJ, 569, 903

[4] Blay, P., Reig, P., Martínez Núnez, S., et al., 2004, A\&A, 427, 293

[5] Bondi, H., Hoyle, F. 1944, MNRAS, 104, 273

[6] Camero Arranz, A., Wilson, C. A., Finger, M. H., Reglero, V., 2007, A\&A, 473, 551

[7] Davidson, K., Ostriker, J. P., 1973, ApJ, 179, 585

[8] Deeter, J. E., Boynton, P. E., Pravdo, S. H., 1981, ApJ, 247, 1003

[9] Galis, R., Beckmann, V., Bianchin, V., Grebenev, S., McBreen, B., Mereghetti, S., Revnivtsev, M., Sanchez, C., Strong, A., Westergaard, N. J., 2007 The Astronomer's Telegram, \#1063

[10] Ghosh, P., Lamb, F. K., 1979, ApJ, 234, 296

[11] Hulleman, F., in 't Zand, J. J. M., Heise, J., 1998, A\&A, 337, L25

[12] İnam, S. Ç., Baykal, A., Swank, J., Stark, M. J., 2004, ApJ, 616, 463

[13] Leahy, D. A., 1987, $A \& A, \mathbf{1 8 0}, 275$

[14] Reig, P., Negueruela, I., Fabregat, J., et al., 2004, A\&A, 421, 673

[15] Reig, P., Negueruela, I., Papamastorakis, G., Manousakis, A., Kougentakis, T., 2005, A\&A, 440, 637

[16] Sidoli, L., Mereghetti, S., Larsson, S., et al., 2005, A\&A, 440, 1033

[17] Vacca, W. D., et al., 1996, The Astrophysical Journal, 460, 914

[18] Waters, L. B. F. M., de Martino, D., Habets, G. M. H. J., Taylor, A. R., 1989, A\&A, 223, 207

[19] Waters, L. B. F. M., van den Heuvel, E. P. J., Taylor, A. R., Habets, G. M. H. J., Persi, P., 1988, A\&A, 198, 200 